\title{
Le Corbaccio en France : imitations et traductions
}

\section{Pauline Pionchon}

\section{OpenEdition \\ Journals}

Édition électronique

URL : http://journals.openedition.org/cei/901

DOI : 10.4000/cei.901

ISSN : 2260-779X

Éditeur

UGA Éditions/Université Grenoble Alpes

\section{Édition imprimée}

Date de publication : 15 juillet 2008

Pagination : 209-223

ISBN : 978-2-84310-122-9

ISSN : $1770-9571$

Référence électronique

Pauline Pionchon, «Le Corbaccio en France : imitations et traductions », Cahiers d'études italiennes [En ligne], 8 | 2008, mis en ligne le 15 janvier 2010, consulté le 01 mai 2019. URL : http:// journals.openedition.org/cei/901 ; DOI : 10.4000/cei.901 


\title{
LE CORBACCIO EN FRANCE
}

\author{
IMITATIONS ET TRADUCTIONS
}

\author{
Pauline Pionchon \\ Université Stendhal-Grenoble 3
}

La dernière édition d'un Corbaccio français, avant celle que les Belles Lettres sont sur le point de publier ${ }^{1}$, remonte à 1788. Il s'agissait alors d'une réimpression de la traduction exécutée en 1698 par un certain Monsieur de Prémont. Plus d'un siècle plus tôt, en 1571, François de Belleforest avait offert au public français la première traduction de cet ouvrage ${ }^{2}$.

Mais il faut garder à l'esprit que les traductions sont bien souvent le point d'arrivée, et non le point de départ, de la fortune d'une œuvre ${ }^{3}$. Avant la première version française, et avant même l'édition française du texte italien, publiée en $1569^{4}$, le Corbaccio est déjà cité par des auteurs français. Dans les ouvrages par lesquels ils prennent part à la querelle des dames, Gratien du Pont et François Billon mentionnent en effet cette œuvre parmi les ouvrages qui blâment les femmes ${ }^{5}$. Dès avant les années soixante du XVI siècle, le Corbaccio avait donc circulé en France.

1. BOCCACE, Corbeau de malheur, traduction de P. Pionchon, préface de G. Baldassarri, Paris, Les Belles Lettres (Bibliothèque italienne), sous presse.

2. Outre ces traductions intégrales, des morceaux choisis ont été publiés en traduction française en 1910 (BOCCACE, Fiammette, Le labyrinthe d'Amour, le Décaméron, Paris, Michaud, 1910). Par ailleurs, Antonio Beccaria réalisa à Londres, dans les années quarante du XVe siècle, une traduction latine du Corbaccio à la demande du comte Humphrey de Gloucester; mais à ce jour on n'a pas trouvé trace d'une quelconque diffusion de ce texte en France (voir Gabriella ALBANESE, «Per la fortuna umanistica di Boccaccio : il Corbaccio latino di Antonio Beccaria", in Studi umanistici, II, 1991, p. 89-150).

3. Lionello SozzI, «Boccaccio in Francia nel Cinquecento", in Il Boccaccio nella cultura francese, éd. C. Pellegrini, Florence, Olschki, 1971, p. 211-349, p. 267.

4. Il Corbaccio, ovvero Laberinto d'Amore, Paris, Federico Morello, 1569.

5. Gratien DU PONT, Controverses des sexes masculin et féminin, Toulouse, 1534, f. CXXVI; François DE BILlon, Fort inexpugnable de l'honneur du sexe féminin, Paris, 1555. 
Il semble que cette œuvre n'ait pas fait l'objet d'imitations. Le projet de la fable autobiographique narrant une conversion de l'intellectuel - dans une lignée à laquelle appartiennent l'Historia calamitatum d'Abélard et le De vetula pseudo-ovidien ${ }^{6}$ ne rencontre plus les inclinations du public. En revanche, le Corbaccio a probablement été utilisé comme un modèle de rhétorique misogyne. Dans la deuxième moitié du XVI siècle, il semble que Jacques Tahureau y ait recouru pour son premier Dialogue ${ }^{7}$, dont le ton et le registre rappellent le livre de Boccace, sans dire que certaines évocations et certains épisodes semblent également tirés du Corbaccio. Il serait d'ailleurs étonnant que, dès le début du siècle, une des nombreuses éditions italiennes ne fût pas tombée entre les mains d'un autre accusateur de l'honneur des dames; tout comme le De mulieribus claris était un modèle obligé de l'elogium mulierum, le Corbaccio a probablement servi assez tôt de réservoir d'arguments pour les champions du camp opposé ${ }^{8}$. Enfin, un grand classique antiféministe du XV siècle, Les XV joyes de mariage, semble également porter un écho du Corbaccio: c'est encore l'éloquence satirique de Boccace qui est exploitée9.

Pour ce qui est des versions françaises, si l'on s'en tient à la période qui va du XIV à la fin du XIX siècle, le Corbaccio n'a été ni plus ni moins traduit que les autres œuvres en langue vulgaire de Boccace, exception faite du Décaméron.

\section{Trois titres pour un livre}

La plus ancienne traduction, celle de Belleforest, porte le titre Le Laberinthe d'Amour, la plus récente, celle de Prémont, Le Songe de Bocace. Ni l'un ni l'autre n'est une traduction littérale du titre italien que Boccace donna à son œuvre: Corbaccio. C'est sous un autre titre que nos traducteurs connaissaient cet ouvrage.

6. Francesco BRUNI, "Historia calamitatum, Secretum, Corbaccio: tre posizioni su luxuria (amor) e superbia (-gloria) ", in IDEM, Testi e chierici del Medioevo, Gênes, Marietti, 1991, p. 203 237; IDEM, "Dal De vetula al Corbaccio: l'idea d'amore e i due tempi dell'intellettuale», in Medioevo romanzo, 2, 1974, p. 161-216.

7. Dialogues de Jacques Tahureau, gentilhomme du Mans, non moins profitables que facétieux, où les vices de chacun sont repris âprement pour nous animer davantage à les fuir et à suivre la vertu. Publié de façon posthume en 1565 , ce texte eut un franc succès, puisqu'il fut réédité quinze fois avant 1602 ; après quoi il tomba dans l'oubli.

8. L. SozzI, «Boccaccio... », cit., p. 268.

9. Ibid., p. 268-269. 
En réalité, ce titre original qui donne du fil à retordre aux savants contemporains, n'était vraisemblablement plus compris au XVI siècle. En effet, certaines copies manuscrites et certaines éditions imprimées portent des sous-titres explicatifs (Corbaccio: il libro del rimedio dello amore, Corbaccio nemico delle femmine, Corbaccius, sive contra sceleratam viduam et alias feminas invectivae $\left.{ }^{10}\right)$. Ailleurs, on trouve le sous-titre - ou tout bonnement le titre - Laberinto d'Amore ${ }^{11}$. C'est une de ces éditions du $X V I^{\mathrm{e}}$ siècle que Belleforest eut entre les mains en $1571^{12}$. L'édition utilisée un siècle plus tard par Prémont portait elle aussi le titre Laberinto d'Amore, comme indique le sous-titre du texte français (ou Labyrinthe d'Amour). En revanche, le titre Le Songe de Bocace est selon toute vraisemblance l'expression de la fantaisie du traducteur. En s'éloignant du titre qu'il croyait original, Prémont eut peut-être l'intention de signifier son éloignement de l'ensemble du texte de Boccace ${ }^{13}$.

\section{Le laberinthe d'Amour de Monsieur Jean Boccace, autrement invective contre une mauvaise femme, mis nouvellement de l'italien en françoys par Monsieur François de Belleforest (1571)}

François de Belleforest (1530-1583) est principalement l'auteur d'ouvrages historiographiques, son activité de traducteur du grec et de l'italien n'étant que secondaire ${ }^{14}$. Ses traductions de Bandello lui valent une place honorable parmi les traducteurs français des classiques italiens. À l'époque où Belleforest travaille à son Corbaccio français ${ }^{15}$, la perception que la France a de Boccace est particulièrement riche.

Au siècle précédent, la France avait connu avant tout les œuvres latines (Genealogie deorum gentilium, De casibus virorum illustrium, De mulieribus

10. Henri Hauvette, «Le Corbaccio, une confession de Boccace», in Bulletin italien, I, 1901, p. 3-21

11. Claudia Sebastiana NobILI, "Per il titolo Corbaccio», in Studi e problemi di critica testuale, XLVIII, 1994, p. 93-114, p. 95. Le syntagme est tiré du texte (\$93).

12. Cette première version française n'a pas pu être élaborée à partir de l'édition française du texte italien de 1569, particulièrement incorrecte contrairement au texte source utilisé, et portant de surcroît les deux titres. Henri HAUVETTE, "Les plus anciennes traductions de Boccace», in Bulletin italien, IX, 1909, p. 1-26, p. 19-20.

13. Voir infra notre étude de la traduction de Prémont.

14. H. HAUVETTE, «Les plus anciennes traductions...», cit., p. 18 - 19.

15. Le laberinthe d'Amour de Monsieur Jean Boccace, autrement invective contre une mavvaise femme, mis nouvellement de l'italien en françoys par Monsieur François de Belleforest, publié par Jean Ruelle, à Paris, en 1571. On peut consulter ce texte à la Bibliothèque de l'Arsenal à Paris. Les pages citées renvoient à la réimpression de 1573. 
claris) de Boccace, qui était perçu surtout comme un savant. Certes, quelques nouvelles circulaient isolément; mais c'était sous le nom d'autres auteurs italiens qui les avaient traduites en latin ${ }^{16}$. Certes, Laurent de Premierfait donna une traduction intégrale du Décaméron (1411-1414); mais celle-là était de plus de dix ans postérieure à celles du De casibus virorum illustrium et du De mulieribus claris, du même traducteur. C'est en tout premier lieu ces deux ouvrages d'érudition qu'il fallait mettre à la portée du public non lettré, et la grande œuvre narrative n'était encore que mineure. Le recensement des manuscrits le prouve: nous possédons une quinzaine de manuscrits du Décaméron de Laurent de Premierfait, contre une soixantaine de son De casibus ${ }^{17}$.

Le XVI e siècle, en revanche, est marqué par une diffusion plus ample et plus profonde d'œuvres jusqu'alors considérées comme mineures ou demeurées inconnues. Boccace est vu comme un homme aux intérêts variés. Les humanistes français continuent à le considérer comme un modèle tutélaire de leurs études. Ils ne mentionnent que brièvement le Décaméron, mais font l'éloge des ouvres latines, qui semblent d'autant plus nombreuses que la paternité d'un nombre certain de textes est attribuée à Boccace indûment ${ }^{18}$. Mais au cours du siècle, ses œuvres vulgaires acquerront de plus en plus de poids. L'atmosphère culturelle de la cour de François Ir et de l'entourage de Marguerite de Navarre y est favorable. Le Ninfale fiesolano, le Filocolo, et surtout la Fiammetta attirent ce public friand de romanesque et de récits d'aventure, qui s'interroge et médite sur l'amour ${ }^{19}$. Par ailleurs, une nouvelle image de Boccace naît de la lecture de ses œuvres vulgaires: celle d'un maître de rhétorique. De sa prose italienne, la langue française doit tirer un style et d'élégantes constructions latinisantes ${ }^{20}$.

En outre, lorsque Belleforest s'atèle au Corbaccio, plusieurs œuvres de Boccace en langue vulgaire ont été récemment traduites: le Décaméron l'a été une nouvelle fois, par Le Maçon, en 1545, le Ninfale fiesolano a été mis en français en 1556, le Filocolo en 1542, l'Elegia di Madonna Fiammetta en 1532 (elle le sera à nouveau en 1582). Sur cette toile de fond, l'idéolo-

16. Je fais référence à la fortune du De insigni obedientia et fide uxoria de Pétrarque (réécriture latine de Dec., X, 10) et de la traduction de Dec. IV, 1 de Leonardo Bruni.

17. Giuseppe Di STEFANO, «Il Trecento», in Il Boccaccio nella cultura francese, cit., p. 1-47.

18. L. SozzI, «Boccaccio...», cit., p. 214-219.

19. Ibid., p. 257-274.

20. Joachim DU BELLAY, Deffence et illustration de la langue française, éd. H. Chamard, Paris, Didier, 1948, p. 75-76; Jean LEMAIRE DE BELGES, La concorde des deux langages, éd. J. Frappier, Genève, Droz, 1947, p. 4. 
gie et la verdeur de ton du Corbaccio promettent de déparer. Dans l'épître à Guy de Quinsay qui fait office de préface, on découvre un traducteur conscient du fait que son Laberinthe d'amour risque de rencontrer un accueil réservé. Il estime néanmoins que l'œuvre mérite d'être « ravie des ténèbres obscures d'oubli où jusqu'à présent elle avait été ensevelie, et cachée», car elle renferme "un vrai labyrinthe de saines interprétations, détournant l'homme de cette furieuse folie d'Amour, et que les hommes suivent comme naturelle, quoi qu' elle soit contre la même perfection de la nature» (f. $2 \mathrm{v}$ ). Ici, Belleforest estime nécessaire de démontrer en quoi une passion aveugle est contre nature, et doit être combattue. Il justifie ainsi la condamnation dont la folie d'amour fait l'objet:

$\mathrm{Vu}$ que l'aimer est naturel, mais que transporter en délices du corps, et s'affectionner plus à ce qui apparait au dehors, qu'à la sincérité de l'âme, ce n'est pas suivre l'inclination naturelle, et propre à l'homme usant de raison, ainsi est se bestialiser, et vautrer en ordures plus sales que celles qui guident le sensuel des animaux incapables de sens, de jugement, et intellect. (f. 2v)

Au prix de quelque violence faite au propos du Corbaccio, Belleforest démontre que la thèse globale de l'œuvre est valable, que la traduction est donc justifiée. Tout comme celle des autres œuvres, la lecture du Corbaccio apporte "plaisir et profit» (f. 3r): le Corbaccio est porteur d'un enseignement moral, son auteur se montre conforme à sa renommée de maître de sagesse. Pour que les éléments de cet enseignement soient facilement accessibles, Belleforest clôt son ouvrage par une «table des choses plus mémorables, et des sentences contenues en ce petit livre».

Un grand embarras parcourt néanmoins de part en part l'épître à Guy de Quinsay. Il transparaît que les propos outrageux prononcés à l'encontre des femmes ne sont plus dans l'air du temps. Les trouver sous la plume de Boccace semble dérouter notre traducteur au point qu'il revient à quatre reprises, dans l'espace de deux feuillets in 18 , sur cette question de la misogynie, qu'il estime être le «sujet» du livre.

Son intervention prend alors plusieurs directions distinctes. D'une part, Belleforest se défend, à deux reprises, d'être l'ennemi des dames, dont il admire au contraire les vertus; les propos qu'il traduit ne sauraient être tenus pour conformes à ses idées. Il s'étonne encore de voir Boccace s'en prendre aux femmes indignes d'être aimées plutôt qu'aux hommes qui s'en entichent brièvement. S'il cautionne le discours sur la passion, le traducteur ne se porte pas garant du discours misogyne. D'autre part, Belleforest tente de défendre l'œuvre qu'il traduit. Il rappellera notamment "quel homme fut le Boccace» (f. 3r), la renommée de l'auteur devant mettre l'œuvre à l'abri du soupçon. Au niveau macroscopique, la défense du Corbaccio repose sur l'enchâssement de la traduction dans un 
dispositif liminaire visant à limiter la portée de l'invective; en guise de préface, l'épître à Quinsay, et comme postface, un sonnet "aux Dames lisant ce livre ${ }^{21} »$. Dans ces deux textes, alors même que le personnage de l'esprit répète à satiété que toutes se valent en infamie, Belleforest précise que Boccace n'entend pas s'en prendre aux vertueuses, mais seulement aux hypocrites de l'espèce de celle que l'ouvrage fustige.

Dans le sonnet qui sert d'envoi, on remarque aussi que Belleforest veut discréditer l'invective. Si Boccace a écrit un texte si virulent contre les femmes, c'est qu'il y fut poussé par l'indignation: une mégère l'avait repoussé et s'était moquée de lui ${ }^{22}$. En somme, il a écrit le Corbaccio sous le coup de la colère, il ne pensait pas ce qu'il écrivait. Son «mécontentement des dames [...] n'est d'ailleurs pas tel qu'on l'estime, en regard à un autre sien œuvre, qui porte le nom des dames illustres» (f. 3r).

Belleforest finit par se désintéresser d'un problème qui lui parait insoluble: il écrit «avoir traduit [le Corbaccio] plus pour la diction que pour le sujet qui y est traité, et plus pour montrer combien Boccace était catholique, que de souci qu'[il a] de son mécontentement des dames» (f. 3r). On a déjà vu que les lettrés français considèrent les œuvres vulgaires de Boccace comme des modèles formels; le Corbaccio fait partie de ce canon $^{23}$. La deuxième raison qu'allègue Belleforest peut étonner davantage. L'invective, qui ne rencontre pas les convictions du traducteur, le plonge certes dans un embarras d'ordre moral; mais il demeure que le Corbaccio se présente comme une ouvre votive ${ }^{24}$ et, dans une certaine mesure, comme une profession de foi. Le champ culturel du Corbaccio contribue à le distinguer des autres ouvres vulgaires de Boccace, des-

21. "Ne faut penser qu'à vous s'attaque ce Boccace,/Et qu'il guerroie l'heur de vos honnêtetés,/Qu'il blâme la valeur de vos pudicités,/Qu'il se moque du doux gisant sous votre grâce./Ne l'estimez si plein d'une orgueilleuse audace/Qu'il égale l'objet des vieilles saletés/Avec la grande splendeur de vos grandes beautés,/Et qu'il blâme en ce lieu seulement qu'une face./Il sait, et je le sais, que le monde est tout plein,/Des femmes qui ont l'heur d'avoir le cœur humain,/L'âme sans fard, le corps vrai miroir de noblesse./Il s'attaque (marri) contre une seulement,/Qui le traita jadis par trop indignement:/Et moi le traduisant aux folles je m'adresse.»

22. Du reste, Henri Hauvette donne à peu près la même interprétation à l'invective plus de trois siècles plus tard («Le Corbaccio...», cit.).

23. Ridolfi mentionne le Corbaccio, à côté du Décaméron et de la Fiammetta, comme une des œuvres utiles à l'apprentissage du bon italien (L.A. RidolFI, Ragionamento avuto in Lione da Claudio de Herberè, gentiluomo francese, e da Alessandro degli Uberti, gentiluomo fiorentino, sopra alcuni luoghi del Cento Novelle del Boccaccio, Lyon, Rouillé, 1557, p. 74). Corbinelli, l'éditeur scientifique de l'édition française de 1569, vante lui aussi les mérites de la langue du Corbaccio dans la lettre qui sert de préface. Voir L. SozzI, «Boccaccio... », cit., p. 268

24. Dans l'incipit, le narrateur annonce que l'œuvre rendra grâce à Dieu et à la Vierge en témoignant d'un bienfait particulier que le poète reçut sans le mériter $(\$ 1-5)$. Nous citons le Corbaccio d'après l'édition par les soins de G. Natali (Milan, Mursia, 1992). 
quelles les problématiques religieuses sont absentes. Or, à une époque profondément marquée par l'esprit scrupuleux de la Réforme catholique, la plus importante de ces œuvres, le Décaméron, subissait les morsures de certains milieux réactionnaires ${ }^{25}$. Il était connu aussi que certaines nouvelles stigmatisaient la corruption de la Curie romaine, les partisans français et italiens d'une réforme de l'Église sachant en faire bon usage ${ }^{26}$. De ce point de vue, et en dépit des problèmes moraux que pose la misogynie qui s'y déploie, le Corbaccio témoigne sinon de la moralité, au moins de l'orthodoxie de Boccace. Belleforest prendra donc le soin d'omettre de sa traduction toutes les phrases irrévérencieuses du point de vue de la religion $^{27}$.

Par ailleurs, ce n'est pas la seule transformation que Belleforest fasse subir à une traduction qui demeure cependant remarquablement fidèle. La syntaxe est généralement calquée sur celle de l'original. On relève quelques contresens; ces contresens, au demeurant rares, dénotent clairement la hâte dans laquelle ce travail a été exécuté28 ${ }^{28}$ "La simple inspection de la série d'œuvres publiées par Belleforest vers 1570-1572 ne laisse aucun doute sur la précipitation avec laquelle il les rédigeait", écrit Hauvette $^{29}$. Dans l'ensemble, Belleforest a une tendance à l'explication et

25. L. SozzI, «Boccaccio... », cit., p. $213-214$.

26. Ibid., p. $220-224$.

27. $\$ 44$ : «Il a un fils de sa femme qui ne lui est rien» («[...] egli ha della moglie un tal figiuolo $[\ldots]$ che gli appartiene meno che a Giuseppe non fece Cristo.»); $\$ 431$ : Belleforest ne traduit pas le commentaire sur les frères qui ont coutume de "secourir" les veuves ("Appropinquossi adunque quanto più poté alla chiesa de'frati $[\ldots]$ acciò che, dove ogni altro uomo le venisse meno, i frati, che santissimi e misericordi uomini sono e consolatori di vedove, non le venissero meno.»); $\$ 250$ : "Ces dames sont si présomptueuses qu'elles se font accroire, pour avoir ouï quelque discours le matin $[\ldots] »$ ("[...] queste, pure una mattina che tanto che una messa si dica stieno alla chiesa, sanno $[\ldots] »)$.

28. Certains sont de moindre importance pour l'intelligence du texte; d'autres occasionnent une distorsion notable du sens : par exemple, $\$ 5($ f. 5 r) : «[...] et qui serve au profit et à la consolation des âmes de ceux qui liront ceci, quoique d'autres, n'en tiendront point de compte.» («[...] e utilità e consolazione dell'anime di coloro li quli per avventura ciò leggeranno, e altro no ". Mais peutêtre que le texte sur lequel Belleforest a travaillé portait «e altri no" ?); $\$ 61$ (f. 16r): Lorsque la brume se dissipe, le poète voit qu’il a été «dévoyé de [son] premier chemin» (Dans le texte italien, le chemin que le poète a suivi l'amène dans le «labyrinthe de l'amour». Dans la traduction, au contraire, le motif perd son sens allégorique.) D’autres aboutissent à des non-sens: $\$ 375$ (f. 90v) : «[...] eussent-ils le visage aussi laid, et difforme que le Sarrazin qui est en la place.» (L'expression " il saracino della piazza " fait référence à un mannequin servant de cible durant les joutes qui se disputaient à Arezzo); $\$ 375$ (f. 91r): L' «Amaroldo d'Irlanda» (le Morholt du cycle de Tristan) devient "l'Admiral d'Irlande». Belleforest n'a pas perdu trop de temps à chercher des équivalents ingénieux: «il fattibello» (\$ 397) e "infornare il malaguida» (\$ 408) sont traduits littéralement le "fais toi belle» et "enfourner le mauvais guide".

29. «Les plus anciennes traductions...", cit., p. 20. 
à l'amplification ${ }^{30}$ : les couples de synonymes sont nombreux ${ }^{31}$. L'amplification sert parfois à expliciter le sens d'un calque de l'italien ${ }^{32}$; mais certaines références, peut-être pas comprises, restent sans autre explication que leur contexte ${ }^{33}$. On relève aussi plusieurs cas d'ajouts entièrement du cru du traducteur ${ }^{34}$, ainsi que des omissions. Celles-ci sont de plusieurs types. Certaines, microscopiques, découlent du délayage pratiqué; d'autres correspondent à une censure du traducteur.

«La pudeur de Belleforest a été offensée par certains passages de l'invective de Boccace, qu'il a librement expurgée en plusieurs endroits", écrit Hauvette ${ }^{35}$. Au vrai, les coupes ne sont pas si nombreuses, et la censure n'a généralement pas été exercée là où on l'attendait. Presque rien n'est omis de l'évocation de la veuve; la description de son corps rebutant est intégralement traduite ${ }^{36}$. En revanche, soucieux de ne pas éclabousser l'ensemble de la gent féminine, Belleforest fait subir au passage traitant des femmes en général des modifications assez profondes eu égard à la diction de l'original, mais à peu près insensibles quant au sens et au ton globaux du passage. En outre, afin d'épargner les vertueuses, il traduit parfois, avec plus ou moins de succès, le substantif générique (mais connoté

30. Voir par exemple: $\$ 6$ (f. $5 \mathrm{v}$ ) : «[...] il m’advint de penser fort profondément sur les accidents de l'amour, et sur les traverses que j'y ai goûtées et savourées en poursuivant les plaisirs de la chair.» («[...] mi avvenne che io fortissimamente sopra gli accidenti del carnale amore cominciai a pensare.»); $\$ 168$ (f. 43v) : «[...] dare legata la mia libertà e sottoposta la mia ragione; e l'anima, che con queste accompagnata soleva essere donna, senza esse divenuta vilissima serva.» («[...] souffrant que la raison qui accompagne mon âme, qui doit être Dame et maitresse, fut une très vile esclave assujetie à l'appétit, et à la convoitise sensuelle [...]».) Belleforest recourt à l'usage d'hyponymes, et insère des adjectifs qualificatifs (voir par exemple $\$ 293$ (f. 72r) : «[...] divenne un serpente $[\ldots]$ "; " une vipère cruelle et dangereuse»).

31. $\$ 52$ (f. 14v): «comme tout repu de viande céleste et sustenté d'un repas divin " («quasi da divino cibo pasciuto »); $\$ 295$ (f. $72 \mathrm{v}$ ) : «[...] j’abaissai la tête et je ployai les épaules [...]» ("piegai le spalle»); $\$ 319$ (f. 78r) : «[...] je sentis mes lèvres tout engluées et empâtées du fard.» ("[...] tutte le labbra m'invischiai $[\ldots] »)$.

32. $\$ 312$ (f. $76 \mathrm{v}$ ): «un autre cinciglion, et grand entonneur de purée de septembre» ("[...] impossibile a credere ti parrebbe di Cinciglion").

33. $\$ 296$ (f. 72v) : "comme si je fusse sorti de Capalle et elle de la race des Rois de Suève" (" [...] come s'io stato fossi da Capalle e ella della casa di Soave [...]») ; $\$ 302$ (f. 75r) : «un Fabrice» ( [ [...] sé d'altra parte di lealtà sopra Fabrizio e qualunque altro leale uomo stato, commendando. ").

34. $\$ 49$ (f. 13v)«[...] avec laquelle [compagnie] me trouvant, et bienviené, et caressé amoureusement de chacun selon que nous en avions bonne coutume toutes les fois que nous nous voyions unis ensemble $[\ldots] »$ ( «[...] colla quale [compagnia] ritrovandomi, e in dilettevole parte raccoltici secondo la nostra antica usanza [...]»); $\$ 48$ (f. 12v-f. 13r) : en traduisant "confortatomi a dover la solitaria dimoranza lasciare, la qual per certo offende molto ciascun il quale della mente è men che sano ", Belleforest brode et évoque le mécontentement et la tristesse des amis du poète absent.

35. «Les plus anciennes traductions...», cit., p. 22.

36. $\$ 308, \$ 309, \S 354, \S 479$ (en partie) ne sont pas traduits. 
péjorativement) femmine par les hyponymes bêtes et folles. Ici il omet un paragraphe entier, là un membre de phrase, par pudeur ou par délicatesse $^{37}$. Ailleurs, il condense quelques phrases, résume un passage ${ }^{38}$. L'ordre du discours s'en trouve parfois bouleversé. Enfin, c'est la verve même du Corbaccio qui a été comme lissée en divers endroits. Dans les petites scènes que les narrateurs évoquent, des détails sont abrégés ou omis, les dialogues sont rapportés en style indirect et écourtés ${ }^{39}$. Ailleurs, la traduction de certains termes est caractérisée par un affaiblissement de l'expressivité, parfois du sens ${ }^{40}$.

Dans l'ensemble, ces corrections ponctuelles n'altèrent pas le sens du texte. Cette traduction n'a rien d'une belle infidèle. Quoique son auteur ne lui consacrât pas un soin extrême, en dépit de faiblesses certaines, elle se montre respectueuse de la lettre comme de l'esprit du Corbaccio. On ne peut dire la même chose de la traduction qu'exécute Monsieur de Prémont à la veille du XVIII siècle.

\section{Le Songe de Bocace, ou le labyrinthe d'Amour, traduit de l'italien par Monsieur de Prémont (1698)}

Comme l'indiquait Hauvette, "ce qu'il y a de trompeur dans ce titre est l'expression traduit de l'italien ${ }^{41} »$. Nous aurons l'occasion de confirmer ce jugement.

Monsieur de Prémont, qui semble posséder une bonne connaissance de la langue et surtout de la littérature italiennes, ne nous est connu que comme l'auteur du Songe de Bocace $e^{42}$. Il exerce une censure sévère sur les

\section{7. $₫ 201, \S 205, \S 221-225$ non traduits.}

38. $\$ 203-204, \$ 206-209, \$ 227-229$ condensés et partiellement expurgés.

39. $\$ 172, \$ 458-459$. Ailleurs, on remarque, comme un repentir, un passage du discours indirect au discours direct: $\$ 333$ (f. 82r) : «[...] ores se plaignant que ce voile n’a pas été assez jauni et ensafrané, ou celui-ci pend trop de ce côté, cet autre est trop haut, retire davantage celui qui me couvre le front, ôte l'épingle que tu m'as mise derrière l'oreille, et mets-la un peu plus loin: ôtemoi ce poil, prend ce verre et racle cette macule, ôte ce petit poil, qui passe plus qu'il ne doit sous l'œil en la joue».

40. Notamment, «la mia bestalità» est traduit par «ma bêtise», qui désignait comme aujourd'hui le manque d'intelligence, et une parole ou un acte sans valeur qui dénote ce manque (voir Dictionnaire Historique de la Langue Française, éd. A. Rey, Paris, Le Robert, 2000³, ad vocem «bête»).

41. «Les plus anciennes traductions...», cit., p. 25.

42. La première édition remonte à 1715 . Le texte est réimprimé pour la dernière fois en 1788 , à Amsterdam ou à Paris, dans le tome XXXI d'une collection intitulée Voyages imaginaires, romanesques, merveilleux, allégoriques, amusants, comiques et critiques, suivis des songes et visions et des romans cabalistiques. La version numérique de cette dernière édition est accessible sur le site de la Bibliothèque nationale de France. 
très nombreuses pages de Boccace qui choquent le bon goût. Cependant, la traduction s'avère beaucoup plus longue que l'original.

J'ai retranché de son ouvrage bien des choses que la pudeur ne souffre point [...]. J'ai remplacé ce que j'ai retranché du texte italien, de contes, de fragments et de vers ${ }^{43}$.

Des textes variés se trouvent insérés dans le corps du texte source expurgé. L'unité originelle est morcelée jusque sur le plan formel, Le Songe de Bocace étant divisé en plusieurs chapitres ${ }^{44}$. Contrairement à ce qu'affirme l'auteur, les récits greffés sur la trame du Corbaccio ne servent pas directement le propos. Il s'agit d'un florilège de textes généralement choisis plus ou moins à propos pour leur prétendue morale misogyne. Celle-ci n'apparaît d'ailleurs souvent qu'au prix d'une manipulation quelque peu malhonnête de la part de Prémont.

Par exemple, c'est assez maladroitement qu'il réoriente Les Deux Procès. Il s'agit d'un récit anonyme en vers, dont voici la trame. Le personnage principal entretient une maîtresse sans se soucier de sa femme, laquelle a elle-même un amant. L'épouse demande l'annulation du mariage pour impuissance du mari, tandis que, tombée enceinte, la mầtresse engage des poursuites contre l'époux. Celui-ci s'amuse de la situation, bien certain qu'il ne pourra perdre les deux procès. C'est pourtant ce qui arrive. Indubitablement, la perfidie féminine est bien illustrée. Toutefois, le pivot du récit est autre: c'est l'absurdité de la double sentence.

Plus loin dans le texte, la visite de la maison de la veuve est prétexte à des citations poétiques tirées d'œuvres absolument étrangères au Corbaccio - du Pastor fido par exemple (p. 155). L'esprit raconte ensuite avoir découvert une lettre "qu'[il] réciter [a] [au poète] pour disposer [son] esprit par cette petite récréation à supporter plus aisément la nouvelle peine qu'[il] lui prépare» (p. 157). Suit une épître à Sappho, chant d'un mari déçu, qui finit de rompre la trame de l'épisode.

Mais le passage le plus débordant d'emprunts à d'autres auteurs est celui où l'esprit évoque comment lui-même et d'autres hommes courtisèrent celle qui allait devenir sa femme (p. 95-103 et p. 110-120). Ce sujet absent du texte originel est longuement développé dans l'adaptation. De

43. Préface, p. xiv-xv. Les citations renvoient à l'édition de 1788.

44. Réflexions sur l'amour, \& sur les malheurs qu'il traîne à sa suite - Commencement du songe de Bocace - Description du Labyrinthe d'Amour - Histoire des amours de Bocace - Belphégor, conte - Les deux procès, conte - Histoire de Griffon \& de la perfide Orgile - Histoire de l'Esprit \& de la Veuve Métamorphose d'Arcante en oranger - Suite de l'histoire de l'Esprit et de sa Veuve - L'esprit fort, conte - Epître à Sapho. 
brefs récits d'amour se succèdent, émaillés de détails rococo ${ }^{45}$, et qui sont autant de prétextes à de petits poèmes de saveur mythologique ou pastorale.

Par ailleurs, l'autobiographisme n'est pas plus mis en doute que dans les textes liminaires de Belleforest. Comme le prouvent le titre Le Songe de Bocace, et ceux de plusieurs chapitres, le personnage du poète est entièrement assimilé à l'auteur. Cette assimilation contamine davantage la traduction que chez Belleforest: on observe notamment que l'esprit s'adresse au poète en le nommant «Pauvre Boccace» (p. 10, p. 163), et que parmi les lectures de la veuve figure "votre Décaméron» (p. 153).

Les innombrables emprunts qui défigurent l'œuvre ont valu à Prémont les foudres de la critique de son époque: à raison, Basnage qualifie l'ouvrage d' " assemblage assez mal assorti du Songe de Bocace et de tout ce que les modernes ont dit longtemps après Boccace sur le chapitre des femmes ${ }^{46} »$. Néanmoins, dans les passages qu'il traduit réellement - relativement brefs en regard de l'ensemble du Songe - Prémont ne s'éloigne pas des principes de traduction de son siècle: il transporte le texte dans l'espace, c'est-à-dire d'une langue à l'autre, puis dans le temps, c'est-à-dire du XIV ${ }^{e}$ au XVII ${ }^{e}$ siècle $^{47}$.

Si l'exposition de l'intrigue est très écourtée, aucun élément de la diégèse ne fait défaut. La syntaxe du texte d'arrivée est absolument dissemblable de celle de l'original: le goût de son temps porte Prémont vers des phrases plus courtes, des constructions plus sobres. Le style du texte français ne réfléchit que rarement celui de Boccace. Prémont préfere les références antiques aux références médiévales. Çà et là, on rencontre quelques références propres au siècle de Prémont; par exemple, l'adjectif «libertin ", qui caractérise la veuve et ses mœurs, ou encore l'évocation d'une ouverture d'opéra sur laquelle le poète aurait composé une chanson qui n'était pas à l'honneur de la veuve (p. 165).

De manière générale, l'adaptation de Prémont déborde de détails inventés par le traducteur. Mis à part une évocation de la veuve occupée à se goinfrer, dans laquelle Prémont se rapproche de la veine «corba-

45. Il est notamment fait mention d' ' une boîte à mouche de vermeil doré, faite en forme de cœur, sur laquelle était gravé un Cupidon".

46. Les savants jugent ce texte ridicule et monstrueux (Pierre BAYLE, in Dictionnaire historique et critique, ad vocem «Boccace», cite H. BASNAGE DE BEAUVAL, Histoire des ouvrages des savants); $\mathrm{cf}$. Giorgio MirandolA, «Il Seicento", in Il Boccaccio nella cultura francese, cit., p. 357-375; Giorgio CERRUTI, «Il Settecento", ibid., p. 377-393, p. 378.

47. G. CERRUTI, «Il Settecento», cit., p. 388-389. 
céenne ${ }^{48}$ ", ses ajouts détonnent. Loin de l'efficacité des touches bien senties de Boccace, ils affaiblissent le ton satirique.

Mais en cette fin de XVII siècle où une traduction collant au texte fait figure d'ouvrage grossier, seule est digne de respect la traduction dite "élégante», qui tient de l'imitation et de la paraphrase. L'auteur médiéval doit être corrigé et adapté au goût contemporain. Les traducteurs ne dédaignent pas l'anachronisme, et s'efforcent de conformer leurs textes aux normes du bon goût ${ }^{49}$ (C'est dire que le projet de traduire le Corbaccio était voué à l'échec!) La traduction dite de Hooge du Décaméron, exécutée et publiée un an avant l'adaptation du Corbaccio, possède ces caractéristiques; le traducteur anonyme «a pris un soin tout particulier de ménager les expressions et d'envelopper les choses de manière que le beau sexe puisse en rire sans rougir» et s'est proposé d'adapter le texte au goût de ses contemporains, auxquels il propose une somme de résumés des différentes nouvelles ${ }^{50}$. Les passages du Songe de Bocace qui sont effectivement traduits de Boccace correspondent à ces normes. L'adoucissement des traits jugés trop crus, les bouleversements infligés à la syntaxe, les condensations, la suppression de l'incipit médiéval ${ }^{51}$, doivent rendre ce texte moderne, plus divertissant et plus actuel. Il demeure que le résultat n'a pas même le privilège de la médiocrité. En dépit de cela, Le songe fut réimprimé dès 1699. La demi-douzaine de rééditions dont cette adaptation fit l'objet au cours du XVIII ${ }^{e}$ siècle prouve qu'elle eut le mérite de bien répondre au goût du siècle à l'aube duquel elle avait été composée.

48. P. 129-130: «S'il y avait au marché quelque bonne pièce de gibier, c'était à quelque prix que ce fût pour la bouche de madame. Les ortolans, les faisans, les petits pieds étaient ses mets ordinaires. Elle n'épargnait pas non plus ces choses dans leur rareté, qu'on fait aux champs les fruits les plus communs dans leur saison. Il n'y avait pour elle ni carême, ni vigile, ni quatre-temps. Si elle mangeait bien, elle ne buvait pas mal. Les vins et les liqueurs paraissaient chez moi sous tant de noms différents, que je n'ai pas eu assez de mémoire pour en retenir la dixième partie. Il lui fallait à chaque repas trois fois plus de vin qu'un honnête homme n'en boit à son ordinaire: elle s'enivrait assez souvent avec des femmes de son humeur. Elles poussaient quelquefois la débauche jusqu'à l'excès: les plus fameux libertins ne l'outrent pas davantage; et dans le temps qu'elle chantait des chansons dissolues, et qu'elle jetait ses coiffures dans le feu, elle s'applaudissait de ses emportements et de ses saillies de bacchante, en disant que la vigne avait été plantée pour elles comme pour nous: qu'elle ne voulait pas se singulariser en ne buvant que de l'eau, ni se priver d'un plaisir que notre fidèle, plus heureux que les autres, commençait à mettre en usage parmi les dames. Si vous aviez vu sa trogne vermeille et entendu son caquet, vous l'auriez prise en ce temps-là pour une folle ou une furie».

49. G. CerRUTI, «Il Settecento», cit., p. 388-389.

50. Les critiques, et les traducteurs postérieurs, condamnent cette traduction (cf. Jean-Pierre Niceron, Mémoires pour servir à l'histoire des hommes illustres de la république des lettres, Paris, 1727-1745, vol. XXXIII). G. CerrUTI, «Il Settecento», cit., p. 390.

51. $\$ 1-5$ non traduits. 
Il faut dire aussi que le Boccace qu'on lit entre XVII et XVIII siècle n'est plus celui de l'époque de Belleforest. Si l'image du maitre de rhétorique perdure (les hommes de lettres considèrent Boccace comme le fondateur de la prose italienne, tandis qu'en Pétrarque ils voient le créateur de la poésie), dès le XVII siècle, Boccace n'est plus en France que l'auteur du Décaméron. Ses autres ouvrages en latin et en italien deviennent des œuvres mineures: parmi ces livres oubliés ou méprisés, seul le Corbaccio jouit du privilège d'une traduction. Pour sa part, le Décaméron est devenu un classique; sa valeur littéraire est une évidence, mais il demeure au centre de polémiques d'ordre moral: Jansénistes, Catholiques et Protestants condamnent une œuvre trop libre ${ }^{52}$. Les traducteurs censurent et corrigent les passages jugés licencieux. Ce sont les aspects riants et galants du Décaméron qui sont mis en avant, et Boccace est désormais perçu comme un auteur de joyeuses bagatelles. Ce profond tournant dans la renommée française de Boccace avait débuté dans les dernières années du XVI siècle: certains auteurs, dont Montaigne, le reléguaient déjà parmi les auteurs "simplement plaisants». Comme le Bandello des nouvelles d'amour et le Piccolomini de l'Historia de duobus amantibus, Boccace était perçu comme le porte-parole d'une éthique galante et libertine ${ }^{53}$. Il est fort probable que face à l'œuvre de cet auteur léger, dont les textes ont peu d'enjeux, le traducteur se soit senti plus libre qu'il ne l'aurait été face à des textes à haute teneur idéologique.

Toutefois, Le songe de Bocace avait besoin d'être présenté dans une introduction qui était en même temps une défense du texte original et de sa traduction. Dans le paratexte de l'édition de 1788, le traducteur et l'éditeur des Voyages apparaissent tiraillés entre deux attitudes opposées. D’une part, ils affirment que le Corbaccio n'a, pas plus que le Décaméron, de teneur idéologique ou de signification morale. La misogynie du discours est d'ailleurs l'effet momentané d'une rancune personnelle ${ }^{54}-$ en somme, comme pour Belleforest, et comme pour Hauvette, l'auteur ne pensait pas ce qu'il écrivait; la portée du propos se trouve réduite, car si Boccace a dépeint une femme mauvaise, il y en eut néanmoins, à chaque époque, de vertueuses, tout comme il y eut des hommes mauvais. Mais d'autre part, de Prémont affirme qu'il s'agit d'une ouvre édifiante: c'est

52. G. Mirandola, «Il Seicento», cit.

53. L. SOZZI, «Boccaccio...», cit., p. 224-225; G. CERRUTI, «Il Settecento», cit., p. 382-384.

54. Soucieux de n'être pas associé aux propos misogynes, Prémont assure n'être "point en colère contre [le sexe féminin], comme Boccace l'était quand il composa son Laberinto d'Amore» (Préface du traducteur, p. xiij). 
avec l'intention de dispenser un enseignement moral que Boccace évoque «les défauts de la plupart des femmes». À la suite d'une expérience douloureuse mais bénéfique, l'écrivain avait souhaité dévoiler aux hommes leurs propres péchés, et les garantir contre ceux de leurs semblables. Il s'agirait donc d'une œuvre véhiculant une idéologie fortement affirmée, et dont les enjeux se situent sur le plan éthique. L'avertissement de l'éditeur est un condensé de ces contradictions:

Le beau sexe nous pardonnera d'employer un ouvrage où il est un peu maltraité, il sait que des invectives de cette nature produites par le dépit ou la jalousie, ne lui ont jamais fait perdre un seul de ses adorateurs. On y reconnaît d'ailleurs la manière légère et badine de l'auteur du Décaméron; c'est de cet ouvrage que Lafontaine a tiré le charmant conte de Belphégor. (p. ix)

L'intention est manifestement de disqualifier l'invective de Boccace. Mais l'éditeur prend aussi le soin de garantir la moralité de l'ouvrage: un expert en sagesse comme La Fontaine en aurait tiré une œuvre - légère comme son modèle. Enfin, elle attache le nom de Boccace à une œuvre en particulier - le Décaméron -, qui place son auteur parmi les écrivains légers. On ne se contente pas de frôler l'absurde.

Les textes introductifs aux deux traductions françaises témoignent, on le voit, de l'embarras que suscite l'idéologie exprimée dans le Corbaccio parmi la postérité. L'image de Boccace qui ressort de cette œuvre semble contredire celle de l'auteur "tendre» et «doux» - ainsi que le qualifia Leonardo Bruni dans ses Vies de Dante et de Pétrarque -, parfois sentimental, en tout cas épris de questions amoureuses, de la Fiammetta et du Décaméron.

Par ailleurs, dans le Corbaccio, un auteur déjà mûr avait donné une forme narrative et satirique à son discours sur la solitude du savant. D'une part, il faut noter que le public concerné par ce discours ne pouvait qu'être réduit (mais y avait-il réellement un public pour entendre ce discours?). D'autre part, cette image toute médiévale du savant décline au début du $\mathrm{XV}^{\mathrm{e}}$ siècle, époque marquée en outre par une revalorisation de la famille et $\mathrm{du}$ rôle de la femme. La réflexion sur les limites de la misogynie, nourrie notamment par les nouvelles traductions latines de la République de Platon et de la Politique d'Aristote, inaugure la querelle des femmes. La violente accusation que prononcent le personnage du poète et celui de l'esprit dans le Corbaccio allait désormais correspondre à une position réactionnaire, ce dont témoignent les textes des éditeurs et des traducteurs. 
Il est certain pour Belleforest, probable pour Prémont, que le prestige de Boccace a été la première motivation pour traduire une ouvre, dont ils ne partageaient pas l'idéologie. Mais le fait que Belleforest n'ait pas censuré les passages les plus crus montre également que ne lui avait pas échappé la belle saveur de cette satire nouvelle, colorée et mordante, que Boccace avait soigneusement forgée sur la base de matériaux classiques et patristiques, mais pour laquelle il s'était aussi profondément inspiré des lettres romanes, et principalement des fabliaux français. 